\title{
ON THE CHANGE OF ROOT NUMBERS UNDER TWISTING AND APPLICATIONS
}

\author{
ARIEL PACETTI
}

(Communicated by Matthew A. Papanikolas)

\begin{abstract}
The purpose of this article is to show how the root number of a modular form changes by twisting in terms of the local Weil-Deligne representation at each prime ideal. As an application, we show how one can, for each odd prime $p$, determine whether a modular form (or a Hilbert modular form) with trivial nebentypus is either Steinberg, principal series or supercuspidal at $p$ by analyzing the change of sign under a suitable twist. We also explain the case $p=2$, where twisting, in general, is not enough.
\end{abstract}

\section{INTRODUCTION}

The theory of local root numbers for modular forms was developed in the wellknown work AL70. If $N=p_{1}^{r_{1}} \cdots p_{t}^{r_{t}}$, for each $i \in\{1, \ldots, t\}$ the authors prove the existence of an involution $W_{p_{i}}$ in the space $S_{k}\left(\Gamma_{0}(N)\right)$ which commutes with the Hecke operators $T_{p}$ (for $p \nmid N$ ) and such that they commute with each other (see their Lemma 8 and Lemma 10). Furthermore, the composition of all such involutions equals the canonical involution $W_{N}$ (see their Lemma 9).

Recall that if $f$ is a modular form in $S_{k}\left(\Gamma_{0}(N)\right)$ which is an eigenfunction for all Hecke operators, its L-series satisfies a functional equation relating its values at $s$ and $1-s$ with a sign factor called the "root number of $f$ ". In the subspace of new forms, the Hecke operators prime to the level diagonalize simultaneously into 1dimensional eigenspaces (the so-called multiplicity one result). Since the canonical involution $W_{N}$ and the Atkin-Lehner involutions $W_{p_{i}}$ commute with such operators, they must leave each eigenspace stable.

If $f$ is a newform, the "local factor of $f$ at $p$ " is the eigenvalue of the involution $W_{p_{i}}$. It can be shown that the root number equals minus the eigenvalue of $W_{N}$ and, in particular, the root number can be read from the local factors.

Besides the definition and properties of the root numbers, the authors also proved some cases of the variance of the local root number under twisting. In particular, the results proven there imply the well-known result that the variance of the global root number of a modular form of level $N$, by twisting by a quadratic field corresponding to a character $\chi$ with a conductor prime to $N$, is given by $\chi(N)$. The case where the level and the conductor are not prime to each other is more subtle. Some partial results were proven in the same work, and some extensions with a similar perspective were obtained in [AL78].

Received by the editors October 20, 2010 and, in revised form, November 8, 2011.

2010 Mathematics Subject Classification. Primary 11F70.

Key words and phrases. Local factors, twisting epsilon factors.

The first author was partially supported by PIP 2010-2012 GI and UBACyT X113.

(C) 2013 American Mathematical Society Reverts to public domain 28 years from publication 
The existence of local factors of representations was proved by Deligne in [Del73]. Many authors used such a description to compute explicitly the local root numbers in terms of the local representation of the Weil-Deligne group (as in GK80 or [Li80]), but to our knowledge, although the variation of the local factor under twisting follows essentially from the properties of the local factor and is known to any expert in the area, it has not been written down explicitly in general. As will be shown in the article, it allows, for example, one to easily compute for an elliptic curve the local type (i.e. whether it is Steinberg, principal series or supercuspidal) at any odd prime (which of course can also be done by looking at the reduction of the elliptic curve, as explained in Roh93, Section 1). For $p=2$, a classification can be given in terms of the sign variation, but this does not completely determine the type (as is shown in Example 4.3 and Example 4.4). If one can have some other information, for example, if one is able to compute the space of modular forms appearing in the quaternion algebra ramified at 2, then this extra information is enough to determine the type at 2 as well.

We should mention the recent article [LW10, where the authors give a method to compute explicitly the local data of a modular form (not just its type) for any nebentypus. They do not use any twisting argument, so their method is different from ours. However, they assume the modular form to be minimal between its twists (and it is not clear how to compute the minimal twist without computing some spaces of smaller levels with any nebentypus; see Example 5.2) and also to get the data they need to compute the whole space of modular forms instead of just the twist of a given one.

By the nature of the argument, the same results hold for Hilbert modular forms with trivial nebentypus as well, but the problem is that in general the global characters to twist by might not exist. Nevertheless, this problem can be overcome by adding some auxiliary prime to the twist, as is shown in the last section, so our method works for Hilbert modular forms as well.

The current article was started some years ago while the author was studying a characterization of the elliptic curves whose conductor is divisible by $p$ but which do not show up in the quaternion algebra ramified at $p$ and at $\infty$. After some numerical computations with Gonzalo Tornaría, we conjectured most of the formulas proven here and applied the formulas to find all elliptic curves in Cremona's tables not appearing in any quaternion algebra (answering a question raised by Professor Cremona to Gonzalo). The table can be found at http://mate.dm.uba. ar/ apacetti/. For a work in progress with Victor Rotger, we needed an exact formula giving the sign variation of a modular form under twisting in terms of the local type, which forced us to reconsider the problem and, by lack of references, write a proof of the conjectures.

Notation. Given an odd prime number $p$, by $\operatorname{val}_{p}(N)$ we denote the $p$-adic valuation of the integer $N$. By $p^{\star}$ we denote the element $\left(\frac{-1}{p}\right) p$, whose square root generates the quadratic extension of $\mathbb{Q}$ ramified only at $p$. For a positive integer $r$, we denote by $U_{r}$ the usual filtration in the $p$-adic units given by

$$
U_{r}=\left\{x \in \mathbb{Z}_{p}^{\times}: x \equiv 1 \quad\left(\bmod p^{r}\right)\right\} .
$$

If $E_{p}$ is a finite extension of $\mathbb{Q}_{p}$, Tr and Norm denote the trace and norm from $E_{p}$ to $\mathbb{Q}_{p}$ respectively. 


\section{Some USEFul WeLL-KNOWN RESUlts}

For $K$ a local field, let $W(K)$ denote the Weil group of $K$, that is, the preimage under the map

$$
\operatorname{Gal}(\bar{K} / K) \mapsto \operatorname{Gal}(\bar{k} / k)
$$

of the integer powers of Frobenius, where $k$ denotes the residue field of $K$. Local class field theory gives an isomorphism between $W(K)^{\mathrm{ab}}$ and $K^{\times}$. Furthermore, if $L / K$ is finite, the following diagrams are commutative:

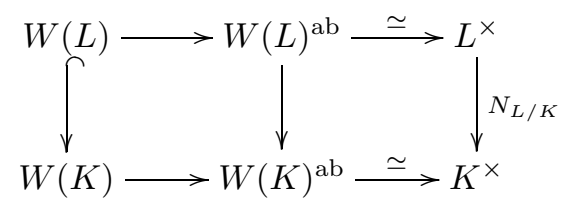

and

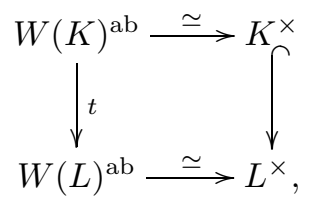

where $t$ denotes the transfer map.

Let $f \in S_{k}\left(\Gamma_{0}(N)\right.$ be a non-zero weight $k$ and level $N$ newform, i.e. a new modular form which is an eigenfunction for all Hecke operators, and let $\rho_{p}(f)$ be the local representation of the Weil-Deligne group $W^{\prime}\left(\mathbb{Q}_{p}\right)$ associated to $f$ at prime $p$. Although it is not so easy to give a description of the Weil-Deligne group (see [Tat79]), it is relatively easy to describe its representations. A complex 2dimensional representation of $W^{\prime}\left(\mathbb{Q}_{p}\right)$ is a pair $(\rho, N)$, where:

(1) $\rho$ is a representation $\rho: W\left(\mathbb{Q}_{p}\right) \mapsto \mathrm{GL}_{2}(\mathbb{C})$,

(2) $N$ is a nilpotent endomorphism of $\mathbb{C}^{2}$ such that

$$
w N w^{-1}=\omega_{1}(w) n, \text { for all } w \in W\left(\mathbb{Q}_{p}\right),
$$

where $\omega_{1}$ is the unramified quasi-character giving the action of $W\left(\mathbb{Q}_{p}\right)$ on the roots of unity (and corresponds to the norm quasi-character $\|\cdot\|_{p}$ under local class field theory).

Although representations of the Weil-Deligne group for any vector space $V$ are defined in a similar way, the two-dimensional complex case is enough for our purposes.

The correspondence between local components of automorphic representations for $\Gamma_{0}(N)$ and the local representations of the Weil-Deligne group is given as follows (using the normalization given by Carayol in [Car86]):

(1) Principal Series (reducible case): The endomorphism $N=0$ and

$$
\rho_{p}(f)=\chi \oplus \chi^{-1} \omega_{1}^{1-k},
$$

for some quasi-character $\chi: W\left(\mathbb{Q}_{p}\right)^{\mathrm{ab}} \mapsto \mathbb{C}^{\times}$.

(2) Steinberg or Special Representation (indecomposable but reducible as a $W\left(\mathbb{Q}_{p}\right)$-representation): The endomorphism $N$ is given by the matrix 
$\left(\begin{array}{ll}0 & 1 \\ 0 & 0\end{array}\right)$, and the representation $\rho_{p}(f)$ is given by

$$
\rho_{p}(f)(w)=\left(\begin{array}{cc}
\chi(w) \omega_{1}(w) & 0 \\
0 & \chi(w)
\end{array}\right),
$$

for some quasi-character $\chi: W\left(\mathbb{Q}_{p}\right) \mapsto \mathbb{C}^{\times}$with $\left.\chi^{2}\right|_{\mathbb{Z}_{p}^{\times}}=1$.

(3) Supercuspidal Representation I (irreducible case, but inertia acts reducibly): The endomorphism $N=0$ and

$$
\rho_{p}(f)=\operatorname{Ind}_{W\left(E_{p}\right)}^{W\left(\mathbb{Q}_{p}\right)} \varkappa,
$$

where $E_{p}$ is a quadratic extension of $\mathbb{Q}_{p}$ and $\varkappa: W\left(E_{p}\right)^{\text {ab }} \mapsto \mathbb{C}^{\times}$is a quasi-character which does not factor through the norm map with a quasicharacter of $W\left(\mathbb{Q}_{p}\right)^{\mathrm{ab}}$ (so that $\rho_{p}(f)$ is irreducible). Furthermore, if $\epsilon_{p}$ denotes the quadratic character corresponding to the extension $E_{p} / \mathbb{Q}_{p}$, then $\epsilon_{p} \varkappa=\|\cdot\|_{p}^{1-k}$ as quasi-characters of $\mathbb{Q}_{p}^{\times}$.

(4) Supercuspidal Representation II (inertia acts irreducibly): This happens only for $p=2$. In this case $N=0$ and the image of $\rho_{p}(f)$ is an exceptional group.

Remark 1 . In the $n$-dimensional case, the last case occurs only for $p \leq n$.

The previous description uses the assumption that the nebentypus of $f$ is trivial, and the third case relies on the following two facts due to Henniart (see Hen79], Theorem 8.2):

Theorem 2.1. Let $E / F$ be a finite separable extension of degree $n$ of local fields and $\rho$ be a linear degree $n$ representations of $W(E)$. If $R$ denotes its induction to $W(F)$, then:

(1) Let $\varepsilon$ be the character of $F^{\times}$that corresponds to the determinant of the permutation representation of $W(F)$ acting on $W(F) / W(E)$. Then for $x \in F^{\times}$one has

$$
\operatorname{det}(R)(x)=\varepsilon(x)^{n} \operatorname{det}(\rho)(x) .
$$

(2) Assume that $\rho$ is semi-simple. Then $R$ is semi-simple as well, and one has

$$
\operatorname{cond}(R)=f(E / F)(n \cdot d(E / F)+\operatorname{cond}(\rho)) \text {, }
$$

where cond () denotes the exponent of the Artin conductor of the representation, $f(E / F)$ denotes the inertial degree and $d(E / F)$ is the discriminant.

\section{THE CASE OF AN ODD PRIME}

The previous description and the fact that the unique order two character of $\mathbb{Z} / p^{r}$ has conductor $p$ give the well-known condition on the exponents of the distinct types:

Corollary 3.1. If $f \in S_{k}\left(\Gamma_{0}(N)\right)$ and $p \neq 2$, we have:

(1) If $\rho_{p}(f)$ is a principal series, then $v_{p}(N)$ is even.

(2) If $\rho_{p}(f)$ is Steinberg, then $\operatorname{val}_{p}(N)=1$ or 2 .

(3) If $\rho_{p}(f)$ is supercuspidal, then $\operatorname{val}_{p}(N) \geq 2$.

Furthermore, in the last case, if $E_{p} / \mathbb{Q}_{p}$ is unramified, then $\operatorname{val}_{p}(N)$ is even. If $E_{p} / \mathbb{Q}_{p}$ is ramified, then $\operatorname{val}_{p}(N)$ is odd unless $\varkappa$ has conductor $1, p \equiv 3(\bmod 4)$ and $\varkappa_{\mathbb{Z}_{p}^{\times}}=\epsilon_{p}$, in which case the conductor is 2 . 
Proof. The first two statements are clear. For the last statement, we know that our representation is the induced representation from a quadratic extension $E_{p}$ of $\mathbb{Q}_{p}$ of a character $\varkappa$. Then Theorem 2.1 implies that since $f$ has trivial nebentypus, we must have

$$
\left.\varkappa\right|_{\mathbb{Z}_{p}^{\times}} \epsilon_{p}=1,
$$

where $\epsilon_{p}$ is the quadratic character that corresponds via class field theory to the quadratic extension $E_{p} / \mathbb{Q}_{p}$. If such an extension is unramified, the conductor of $\varkappa$ must be non-zero, since otherwise it will factor through the norm map. Hence the even condition in the exponent comes from the fact that the inertial degree is 2 in this case.

In the case where the extension is ramified, $d\left(E_{p} / \mathbb{Q}_{p}\right)=1$. Hence Theorem 2.1 implies that the conductor of the representation equals $1+\operatorname{cond}(\varkappa)$.

The conductor of $\epsilon_{p}$ is 1 , so condition (33) implies that cond $(\varkappa)=1$ and $\varkappa_{\mathbb{Z}_{p}^{\times}}=\epsilon_{p}$ or its conductor is even, which implies the statement. Note that in the first case, $\left.\varkappa\right|_{\mathbb{Z}_{p}^{\times}}$is quadratic, and it factors through the norm map if and only if there is a character of order 4 in $\mathbb{Z}_{p}^{\times}$with conductor $p$. This is indeed the case if and only if $p \equiv 1(\bmod 4)$, so the representation is irreducible only when $p \equiv 3(\bmod 4)$.

Remark 2 . If the form $f$ has CM by the extension $\mathbb{Q}[\sqrt{-p}]$, then its local component at $p$ corresponds exactly to $E_{p} / \mathbb{Q}_{p}$ being ramified and $\varkappa$ of conductor 1 .

Let $\chi$ be the quadratic character associated to the quadratic extension of $\mathbb{Q}$ ramified only at $p$. By class field theory, it can be identified with a character of the idèle group, i.e. characters $\left\{\chi_{q}\right\}_{q}$, with $\chi_{q}: \mathbb{Q}_{q}^{\times} \mapsto \mathbb{C}^{\times}$satisfying the following conditions:

- If $q \neq p$, then $\chi_{q}$ is unramified, and $\chi_{q}(q)=\left(\frac{q}{p}\right)$.

- $\chi_{p}$ is ramified with conductor $p$, and its value in $\mathbb{Z}_{p}^{\times}$factors through the unique quadratic character of $\mathbb{F}_{p}^{\times}$. Furthermore, $\chi_{p}(p)=1$.

Given a modular form $f$ in $S_{k}\left(\Gamma_{0}(N)\right)$, we want to study how the local factors of $f$ change while twisting by $\chi$. Denote by $\varepsilon_{q}$ the variation of the local factor of $f$ at $q$ while twisting by $\chi_{q}$, where we choose the same additive character and Haar measure on both computations.

Remark 3. In the correspondence between automorphic forms and representations of the Weil-Deligne group, twisting an automorphic representation by a quasicharacter has the effect of twisting the Weil-Deligne representation by the inverse of the quasi-character, but since our character $\chi_{p}$ is quadratic, we can avoid this technical detail.

Theorem 3.2. The number $\varepsilon_{q}$ is given by:

(1) If $q \neq p$, then $\varepsilon_{q}=\left(\frac{q}{p}\right)^{\operatorname{val}_{q}(N)}$.

(2) If $\rho_{p}(f)$ is principal series, then

$$
\varepsilon_{p}= \begin{cases}\left(\frac{-1}{p}\right) & \text { if } \operatorname{val}_{p}(N) \neq 0 \\ \left(\frac{-1}{p}\right) p^{k} & \text { if } \operatorname{val}_{p}(N)=0 .\end{cases}
$$

(3) If $\rho_{p}(f)$ is supercuspidal and $E_{p} / \mathbb{Q}_{p}$ is unramified, then $\varepsilon_{p}=-\left(\frac{-1}{p}\right)$. 
(4) If $\rho_{p}(f)$ is supercuspidal and $E_{p} / \mathbb{Q}_{p}$ is ramified, then

$$
\varepsilon_{p}= \begin{cases}1 & \text { if } \operatorname{val}_{p}(N)=2 \\ 1 & \text { if } E_{p}=\mathbb{Q}_{p}\left[\sqrt{p^{\star}}\right] \\ -1 & \text { elsewhere. }\end{cases}
$$

(5) If $\rho_{p}(f)$ is Steinberg with $\operatorname{val}_{p}(N)=1$, choosing the additive character $\psi$ unramified and the Haar measure normalized such that $\int_{\mathbb{Z}_{p}} d x=1$, the local sign is given by

$$
\varepsilon\left(\rho_{p}(f), \psi, d x\right)=\frac{-1}{\chi(p)},
$$

while the local sign of $\rho_{p}(f) \otimes \chi_{p}$ is given by

$$
\varepsilon\left(\rho_{p}(f) \otimes \chi_{p}, \psi, d x\right)=\left(\frac{-1}{p}\right) .
$$

Remark 4. Although in the second case the local root number is not just a sign, the power of the prime $p$ appearing comes from the fact that the level of the form and its twists are different.

Remark 5. The result for the Steinberg representation and for the principal series when $p \nmid N$ is well known and can be found for example in AL70. (Lemma 30 and Theorem 6), although the way to prove it uses the theory of the Atkin-Lehner involutions as global actions, while the proof we present is just of a local nature.

The proof of the result is quite elementary and is mainly based on the properties that the local constant satisfies, as explained in Del73. One of the main properties that determines the local constant is the following:

Property. Let $\rho$ be a virtual 0-dimensional representation of a finite extension $E_{p} / \mathbb{Q}_{p}$. Then

$$
\varepsilon\left(\operatorname{Ind}_{W\left(E_{p}\right)}^{W\left(\mathbb{Q}_{p}\right)} \rho, \psi\right)=\varepsilon\left(\rho, \psi \circ \operatorname{Tr}_{E_{p} / \mathbb{Q}_{p}}\right)
$$

See Del73, Theorem 4.1, for a proof of the existence of local constants with the appropriate 4 conditions.

Proof of Theorem 3.2. We consider each case separately:

(1) If $q \neq p$, the character $\chi_{q}$ is unramified; hence by (5.5.1) of Del73,

$$
\varepsilon\left(\rho_{q}(f) \otimes \chi_{q}, \psi, d x\right)=\chi_{q}\left(q^{\operatorname{val}_{q}(N)+q \operatorname{dim}\left(\rho_{q}(f)\right)}\right) \varepsilon\left(\rho_{q}(f), \psi, d x\right) .
$$

Since $\chi_{q}(q)=\left(\frac{q}{p}\right)$ and $\operatorname{dim}\left(\rho_{q}(f)\right)=2$, the statement follows.

(2) Since $\rho_{p}(f)$ is reducible in the principal series case, we need to see how the local constant of a quasi-character changes under twisting by $\chi_{p}$. Let $a=\operatorname{cond}\left(\chi_{1}\right)$ be the conductor of the quasi-character $\chi_{1}$ and choose $\psi$ to be an additive character of $\mathbb{Q}_{p}$ with $\operatorname{cond}(\psi)=0$ (i.e. $\left.\psi\right|_{\mathbb{Z}_{p}}=1$, but $\left.\psi\right|_{\frac{1}{p} \mathbb{Z}_{p}} \neq 1$ ) and the Haar measure $d x$ such that $\int_{\mathbb{Z}_{p}} d x=1$. The local epsilon factor is then given by

$$
\int_{\mathbb{Z}_{p}^{\times}} \chi_{1}^{-1}\left(\frac{x}{p^{a}}\right) \psi\left(\frac{x}{p^{a}}\right) d \frac{x}{p^{a}}=\chi_{1}(p)^{a} p^{a} \sum_{b \in \mathbb{Z}_{p}^{\times} / U_{a}} \chi_{1}^{-1}(b) \psi\left(\frac{b}{p^{a}}\right) \int_{U_{a}} d x
$$


The normalization $\int_{\mathbb{Z}_{p}} d x=1$ implies that $\int_{\mathbb{Z}_{p}^{\times}} d x=\frac{p-1}{p}$ and $\int_{U_{a}} d x=\frac{1}{p^{a}}$. Since the conductor of $\psi$ is $0, \psi\left(\frac{1}{p^{a}}\right)=\exp \left(2 \pi i / p^{a}\right)^{c}$ for some $c$ prime to $p$. Then

$$
G\left(\chi_{1}^{-1}, c\right)=\chi_{1}^{-1}(c)\left(\sum_{b \in \mathbb{Z}_{p}^{\times} / U_{a}} \chi_{1}^{-1}(b) \exp \left(\frac{2 \pi i b}{p^{a}}\right)\right)
$$

is a Gauss sum. If we compute the product of the epsilon factor corresponding to $\chi_{1}$ and the one corresponding to $\chi_{1}^{-1}\|\cdot\|_{p}^{1-k}$, we get that the local factor is given by

$$
\left\|p^{a}\right\|_{p}^{1-k} G\left(\chi_{1}^{-1}, c\right) G\left(\chi_{1}, c\right)=\frac{p^{a k}}{p^{a}} p^{a} \chi_{1}(-1)=p^{a k} \chi_{1}(-1) .
$$

The middle equality is a classical result of Gauss sums; see for example Dav00] (Ex. 13(9), p. 295). If we replace $\chi_{1}$ by $\chi_{1} \chi_{p}$ in the previous computation, we get that the two numbers differ by $\chi_{p}(-1)=\left(\frac{-1}{p}\right)$ and a power of $p$ if the level of $f$ and that of $f \otimes \chi$ are not equal, as claimed.

(3) Since $\left(\operatorname{Ind}_{W\left(E_{p}\right)}^{W\left(\mathbb{Q}_{p}\right)} \varkappa\right) \chi_{p}=\operatorname{Ind}_{W\left(E_{p}\right)}^{W\left(\mathbb{Q}_{p}\right)}\left(\chi_{p} \varkappa\right)$ (where in the second term of the equality we are considering the restriction of $\chi_{p}$ to $\left.W\left(E_{p}\right)\right)$, we can apply the Property stated before to $\rho=\chi_{p} \varkappa-\varkappa$. Then

$$
\frac{\varepsilon\left(\operatorname{Ind}_{W\left(E_{p}\right)}^{W\left(\mathbb{Q}_{p}\right)} \chi_{p} \varkappa, \psi\right)}{\varepsilon\left(\operatorname{Ind}_{W\left(E_{p}\right)}^{W\left(\mathbb{Q}_{p}\right)} \varkappa, \psi\right)}=\frac{\varepsilon\left(\chi_{p} \varkappa, \psi \circ \operatorname{Tr}_{E_{p} / \mathbb{Q}_{p}}\right)}{\varepsilon\left(\varkappa, \psi \circ \operatorname{Tr}_{E_{p} / \mathbb{Q}_{p}}\right)} .
$$

This allows us to restrict to the 1-dimensional case. Recall that since $E_{p} / \mathbb{Q}_{p}$ is unramified, $\varkappa$ is ramified (as was pointed out in the proof of Corollary 3.1). Let $\mathscr{O}_{p}$ denote the ring of integers of $E_{p}$, take $p$ as a local uniformizer, and let $a=\operatorname{cond}(\varkappa)$ be the conductor of $\varkappa$. Recall that since the nebentypus of $f$ is trivial, $\left.\varkappa\right|_{\mathbb{Z}_{p}^{\times}}=1$. Let $\psi$ be an additive character with $\operatorname{cond}(\psi)=0$ as before, and $d x$ a Haar measure such that $\int_{\mathscr{O}_{p}} d x=1$. To ease notation we will denote by $\tilde{\psi}$ the additive character $\psi \circ \operatorname{Tr}_{E_{p} / \mathbb{Q}_{p}}$. The local factor is given by

$$
\begin{aligned}
\varepsilon(\varkappa, \tilde{\psi}, d x)=\int_{\mathscr{O}_{p}^{\times}} \varkappa^{-1}\left(\frac{x}{p^{a}}\right) \tilde{\psi} & \left(\frac{x}{p^{a}}\right) d \frac{x}{p^{a}} \\
& =\varkappa(p)^{a} p^{2 a}\left(\sum_{b \in \mathscr{O}_{p}^{\times} / U_{a}} \varkappa^{-1}(b) \tilde{\psi}\left(\frac{b}{p^{a}}\right)\right) \int_{U_{a}} d x .
\end{aligned}
$$

The middle sum can be written as

$$
\begin{aligned}
\sum_{\alpha \in\left(\mathscr{O}_{p} / p^{a}\right)^{\times} /\left(\mathbb{Z}_{p} / p^{a}\right) \times} \sum_{\beta \in\left(\mathbb{Z}_{p} / p^{a}\right)^{\times}} \varkappa^{-1}(\alpha \beta) \psi\left(\frac{\operatorname{Tr}(\alpha) \beta}{p^{a}}\right) \\
=\sum_{\alpha} \varkappa^{-1}(\alpha) \sum_{\beta} \psi\left(\frac{\operatorname{Tr}(\alpha) \beta}{p^{a}}\right) .
\end{aligned}
$$

If $p^{a-1} \nmid \operatorname{Tr}(\alpha)$, the last sum is zero, since it is a sum over all primitive roots of unity of order at least $p^{2}$. For elements where $p^{a-1}|| \operatorname{Tr}(\alpha)$ (i.e. $p^{a-1} \mid \operatorname{Tr}(\alpha)$ but $\left.p^{a} \nmid \operatorname{Tr}(\alpha)\right)$, the last sum is -1 . Such elements are of the form

$$
r\left(p^{a-1}+\beta \sqrt{\delta}\right), \quad r \in\left(\mathbb{Z}_{p} / p^{a}\right)^{\times} \text {and } p \nmid \beta .
$$


Modulo multiplication by elements of $\left(\mathbb{Z}_{p} / p^{a}\right)^{\times}$in $\mathscr{O}_{p} / p^{a}$, for $p \nmid s$, we have that

$$
\left(p^{a-1}+\beta \sqrt{\delta}\right)^{s} \sim\left(p^{a-1}+s^{-1} \beta \sqrt{\delta}\right),
$$

so the sum with these terms is

$$
(-1) \cdot \sum_{p^{a-1}|| \operatorname{Tr}(\alpha)} \varkappa^{-1}(\alpha)=(-1) \cdot \sum_{s \in\left(\mathbb{Z}_{p} / p^{a}\right)^{\times}} \varkappa^{-1}\left(p^{a-1}+\sqrt{\delta}\right)^{s} .
$$

Since the conductor of $\varkappa$ is $p^{a}, \varkappa$ is non-trivial on such elements and the last sum is zero. Then the only remaining terms are the ones with $\operatorname{Tr}(\alpha)=0$ (i.e. $\alpha=\sqrt{\delta}$ for a non-square element $\delta$ ), and in this case all terms of the last sum are 1 so we get that

$$
\varepsilon(\varkappa, \tilde{\psi}, d x)=p^{a-1}(p-1) \varkappa(\sqrt{\delta})^{-1} \varkappa(p)^{a},
$$

where $\delta$ is a non-square in $\mathbb{Q}_{p}$. If we make the same computation with $\chi_{p} \varkappa$, we get that

$$
\varepsilon_{p}=\frac{p^{a-1}(p-1) \varkappa(\sqrt{\delta})^{-1} \chi_{p}(-\delta) \varkappa(p)^{a}}{p^{a-1}(p-1) \varkappa(\sqrt{\delta})^{-1} \varkappa(p)^{a}}=-\left(\frac{-1}{p}\right) .
$$

(4) This case is similar to the previous one, with the main difference being that by using the commutative diagram (11), we need to compose our character with the norm map. This gives another character (that by abuse of notation we also denote $\left.\chi_{p}\right)$ which satisfies $\left.\chi_{p}\right|_{\mathscr{O}_{p}^{\times}}=1$, because the conductor of $\chi_{p}$ is $p$ and the norm map from $\mathscr{O}_{p}^{\times}$to $\mathbb{Z}_{p}^{\times}$gives only squares modulo $p$. Then the terms in the sum are the same for $\varkappa$ and $\chi_{p} \varkappa$. Since $E_{p} / \mathbb{Q}_{p}$ is ramified, the conductor of $\psi \circ \operatorname{Tr}=1$; hence if we take $\pi=\sqrt{p \delta}$ as a local uniformizer, the local factor is given by

$$
\varepsilon(\varkappa, \tilde{\psi}, d x)=\varkappa\left(\pi^{\operatorname{cond}(\varkappa)+1}\right) \int_{\mathscr{O}_{p}^{\times}} \varkappa^{-1}(x) \tilde{\psi}\left(\frac{x}{\pi^{2 r+1}}\right) d \frac{x}{\pi^{2 r+1}} .
$$

The local factor of the twisted representation is given by

$$
\varepsilon\left(\tilde{\chi}_{p} \varkappa, \tilde{\psi}, d x\right)=\chi_{p}(\operatorname{Norm}(\pi))^{\operatorname{cond}(\varkappa)+1} \varepsilon(\varkappa, \tilde{\psi}, d x) .
$$

Hence the quotient equals 1 if $\operatorname{cond}(\varkappa)=1$ or

$$
\varepsilon_{p}=\left(\frac{\operatorname{Norm}(\pi) / p}{p}\right)=\left(\frac{-\delta}{p}\right) .
$$

In particular, if $\delta=\left(\frac{-1}{p}\right)$ up to squares, $\varepsilon_{p}=1$; while if it does not, then $\varepsilon_{p}=-1$, as claimed.

(5) This comes from the definition of the local epsilon factor attached to the representations where the nilpotent endomorphism is not trivial and how the local epsilon factors change for the principal series.

Let $f$ be in $S_{k}\left(\Gamma_{0}(N)\right)$, where $N=p^{r} N^{\prime}$, with $p \nmid N$, and let $\varepsilon(f)$ be its functional equation sign. Let $\chi_{p}$ be as before, and let $f \otimes \chi_{p}$ denote the newform obtained while twisting $f$ by $\chi_{p}$. Denote its level by $N\left(f \otimes \chi_{p}\right)$. The previous statement allows the following classification:

Corollary 3.3. With the previous notation, we have the following computational criteria to compute the local type:

- $\pi_{p}(f)$ is Steinberg if $\operatorname{val}_{p}(N)=1$ or $\operatorname{val}_{p}\left(N\left(f \otimes \chi_{p}\right)\right)=1$. 
- $\pi_{p}(f)$ is principal series if it is not Steinberg, $2 \mid \operatorname{val}_{p}(N)$ and

$$
\varepsilon\left(f \otimes \chi_{p}\right)=\chi_{p}\left(N^{\prime}\right) \varepsilon(f)\left(\frac{-1}{p}\right) .
$$

- $\pi_{p}(f)$ is supercuspidal if it is not of the above type. Furthermore, if $\operatorname{val}_{p}(N)$ is even and greater than $2, \pi_{p}(f)$ is induced from the unramified quadratic extension of $\mathbb{Q}_{p}$, while if $\operatorname{val}_{p}(N)$ is odd and greater than 2 ,

- $\pi_{p}(f)$ is induced from the extension $\mathbb{Q}_{p}\left[\sqrt{p^{\star}}\right]$ if $\varepsilon\left(f \otimes \chi_{p}\right)=\chi_{p}\left(N^{\prime}\right) \varepsilon(f)$.

- $\pi_{p}(f)$ is induced from the extension $\mathbb{Q}_{p}\left[\delta \sqrt{p^{\star}}\right]$ (for any non-square $\delta$ ) if $\varepsilon\left(f \otimes \chi_{p}\right)=-\chi_{p}\left(N^{\prime}\right) \varepsilon(f)$.

Remark 6. In the case $\operatorname{val}_{p}(N)=2$, twisting only allows us to determine the type, but does not distinguish from which quadratic extension the representation is induced.

Remark 7 . We can replace the global functional equation sign in the last two corollaries by the local Atkin-Lehner involution $W_{p}$. Then the same statements are true by replacing $\varepsilon()$ with the eigenvalue of $W_{p}$ and removing the factor $\chi_{p}\left(N^{\prime}\right)$.

Remark 8. If $f \in S_{k}\left(\Gamma_{1}(N), \epsilon\right)$ and for $p \mid N$ the character $\epsilon_{p}=1$, the same result holds.

\section{THE CASE $p=2$}

When $p=2$, there are more representations of the Weil group. It is a classical result that all subgroups of $\mathrm{PGL}_{2}(\mathbb{C})$ are isomorphic to a cyclic group, a dihedral group, $A_{4}, S_{4}$ or $A_{5}$. The $A_{5}$ case cannot happen since the Galois group $\operatorname{Gal}\left(\overline{\mathbb{Q}}_{p} / \mathbb{Q}_{p}\right)$ is solvable.

The case $p \neq 2$ did not include exceptional cases since for odd characteristic all 2-dimensional representations of $W\left(\mathbb{Q}_{p}\right)$ are of dihedral type as mentioned in Remark 1. But for $p=2$, the $A_{4}$ and $S_{4}$ cases might occur. Weil proved in Wei74] that over $\mathbb{Q}$, the $A_{4}$ case actually does not occur, so the only exceptional case has image $S_{4}$. Furthermore, there are only 8 cases with projective image $S_{4}$ and in all cases it corresponds to the field extension of $\mathbb{Q}_{2}$ obtained by adding the coordinates of the 3-torsion points of the elliptic curves (see also [BR99], Section 8):

$$
E_{1}^{(r)}: r y^{2}=x^{3}+3 x+2, \quad r \in\{ \pm 1, \pm 2\}
$$

and

$$
E_{2}^{(r)}: r y^{2}=x^{3}-3 x+1, \quad r \in\{ \pm 1, \pm 2\}
$$

Note that there are three quadratic extensions of $\mathbb{Q}$ which ramify only at 2 . We will denote by $\chi_{-1}, \chi_{2}$ and $\chi_{-2}$ the quadratic character that corresponds to such quadratic extensions, where $\chi_{i}$ corresponds to $\mathbb{Q}[\sqrt{i}]$ (the first one of conductor 4 and the last two of conductor 8). Then the four curves of each type are twists of each other, where $E_{i}^{(r)} \otimes \chi_{j}=E_{i}^{(r j)}$ (abusing notation and considering the supraindices modulo the equivalence relation given by squares). Furthermore, by Rio06, Section 6], the level of the modular form is $2^{7}$ in the case of the curve $E_{1}^{(r)}$ (with $r \in\{ \pm 1, \pm 2\}), 2^{4}$ for the curve $E_{2}^{(1)}, 2^{3}$ for the curve $E_{2}^{(-1)}$ and $2^{6}$ for the curve $E_{2}^{( \pm 2)}$.

Before stating the equivalent of Corollary 3.1, recall that there are seven quadratic extensions of $\mathbb{Q}_{2}$. One of them is unramified, two of them have a discriminant 
with valuation 2 (corresponding to $\sqrt{3}$ and $\sqrt{7}$ ), and four of them have a discriminant with valuation 3 (corresponding to $\sqrt{2}, \sqrt{10}, \sqrt{-2}$ and $\sqrt{-10}$ ). With the previous notation, we have

Corollary 4.1. For $p=2$, we have:

- If $\rho_{2}(f)$ is a principal series, then $v_{2}(N)$ is even (but not 2$)$.

- If $\rho_{2}(f)$ is Steinberg, then $\operatorname{val}_{2}(N) \in\{1,4,6\}$.

- If $\rho_{2}(f)$ is supercuspidal, then $\operatorname{val}_{2}(N) \geq 2$. Furthermore, depending on the different extensions we have:

- If $E_{2} / \mathbb{Q}_{2}$ is unramified, then $\operatorname{val}_{2}(N)$ is even and greater than or equal to 2 .

- If $E_{2} / \mathbb{Q}_{2}$ is ramified with valuation 2 , then $\operatorname{val}_{2}(N)=5$ or it is even and greater than or equal to 6 .

- If $E_{2} / \mathbb{Q}_{2}$ is ramified with valuation 3 , then $\operatorname{val}_{2}(N)=8$ or it is odd and greater than or equal to 9 .

- If $\rho_{2}(f)$ is supercuspidal of type II, then $\operatorname{val}_{2}(N) \in\{3,4,6,7\}$.

Proof. The proof is the same as before for the first two cases. The supercuspidal dihedral case is also the same for the unramified extension, while for the ramified extension, note that if $\left.\varkappa\right|_{\mathbb{Z}_{2}^{\times}}=\epsilon_{2}$, then the conductor of $\varkappa$ is $2 \cdot \operatorname{cond}\left(\epsilon_{2}\right)-1$ or it is even (and greater). Then Theorem 2.1 implies that the conductor of the induced representation is $3 \cdot \operatorname{cond}\left(\epsilon_{2}\right)-1$ or congruent to cond $\left(\epsilon_{2}\right)$ modulo 2 .

Remark 9. Note that in the supercuspidal dihedral case induced from ramified quadratic extensions, all the representations are irreducible, since there are no characters of order 4 and conductor 4 nor characters of order 4 and conductor 8 .

Remark 10. In the principal series case, the levels 1,4 and 6 are twists of each other, since all characters of conductor 1,2 or 3 are at most quadratic.

We summarize the previous corollary in Table 4.1 (where we used the notation: PS meaning principal series; ST meaning Steinberg; SCI $a$, SCI $b$, SCI $c$ meaning supercuspidal of dihedral type; the index $a, b$ of $c$ meaning induced from an extension with discriminant 0,2 and 3 , respectively; and SCII meaning supercuspidal of second type).

TABle 4.1. Possible types for $p=2$.

\begin{tabular}{|c|c|c|c|}
\hline $\operatorname{val}_{2}(N)$ & Types & $\operatorname{val}_{2}(N)$ & Types \\
\hline \hline 0 & PS & 6 & PS, ST, SCI $a$, SCI $b$, SCII \\
\hline 1 & ST & 7 & SCII \\
\hline 2 & SCI $a$ & 8 & PS, SCI $a$, SCI $b$, SCI $c$ \\
\hline 3 & SCII & odd $\geq 9$ & SCI $c$ \\
\hline 4 & PS, ST, SCI $a$, SCII & even $\geq 10$ & PS, SCI $a$, SCI $b$ \\
\hline 5 & SCI $b$ & & \\
\hline
\end{tabular}

Let $\chi$ denote the character associated by class field theory to any of the characters $\chi_{i}, i \in\{-1, \pm 2\}$, and denote by $\varepsilon_{q}$ the change of the variation of the local factor at $q$ under twisting by $\chi$. Then $\varepsilon_{q}$ it is given by: 
Theorem 4.2. The number $\varepsilon_{q}$ (up to a power of 2 ) is given by:

(1) If $q \neq 2$, then $\varepsilon_{q}=\chi(q)^{\operatorname{val}_{q}(N)}$.

(2) If $\rho_{2}(f)$ is principal series, then $\varepsilon_{2}=\chi(-1)$.

(3) If $\rho_{2}(f)$ is supercuspidal and $E_{2} / \mathbb{Q}_{2}$ is unramified, then $\varepsilon_{2}=-\chi(-1)$.

(4) If $\rho_{2}(f)$ is supercuspidal and $E_{2} / \mathbb{Q}_{2}$ is ramified, then

$$
\varepsilon_{2}= \begin{cases}1 & \text { if } \operatorname{cond}(\varkappa)=2 \cdot \operatorname{cond}\left(\epsilon_{2}\right)-1, \\ 1 & \text { if } E_{2} \text { corresponds to } \chi_{2}, \\ -1 & \text { elsewhere. }\end{cases}
$$

(5) If $\rho_{2}(f)$ is Steinberg with $\operatorname{val}_{2}(N)=1$, choosing the additive character $\psi$ unramified and the Haar measure normalized such that $\int_{\mathbb{Z}_{2}} d x=1$, the local sign is given by

$$
\varepsilon\left(\rho_{2}(f), \psi, d x\right)=\frac{-1}{\chi(2)},
$$

while the local sign of $\rho_{2}(f) \otimes \chi_{2}$ is given by

$$
\varepsilon\left(\rho_{2}(f) \otimes \chi_{2}, \psi, d x\right)=\chi_{2}(-1) .
$$

Proof. The proof is almost the same as in the odd case and can easily be checked.

Remark 11. The computation in the non-dihedral supercuspidal case is straightforward, since one can compute for each elliptic curve the local root number and use the relations between the curves under twisting. In Table 4.2 we list the local root numbers of each curve at 2 .

TABLE 4.2. Root numbers in the non-dihedral supercuspidal case.

\begin{tabular}{|c|r|c|r|}
\hline Curve & Root Number & Curve & Root Number \\
\hline \hline$E_{1}^{(1)}$ & 1 & $E_{2}^{(1)}$ & 1 \\
\hline$E_{1}^{(-1)}$ & 1 & $E_{2}^{(-1)}$ & 1 \\
\hline$E_{1}^{(2)}$ & -1 & $E_{2}^{(2)}$ & -1 \\
\hline$E_{1}^{(-2)}$ & 1 & $E_{2}^{(-2)}$ & 1 \\
\hline
\end{tabular}

Remark 12. Contrary to the odd case, where the variation of the sign under twisting allows us to compute the exact type of representation, for $p=2$ this is no longer the case. The only cases that cannot be distinguished are those of a principal series representation and a dihedral supercuspidal representation induced from the quadratic extension $\mathbb{Q}_{2}(\sqrt{2})$; in this case they are not twists of lower level, so in particular $\operatorname{val}_{2}(N)$ is even and greater than 8 . This is so by Theorem 4.2 since, following the previous notation, $\chi_{-1}(-1)=-1, \chi_{-2}(-1)=-1$ and $\chi_{2}(-1)=1$.

Example 4.3. Consider the curve $E 768 b$ in Cremona's notation. Its quadratic twists by $\chi_{-1}, \chi_{2}$ and $\chi_{-2}$ are the curves E768h, E768d and E768f respectively (an online table of the curves and their first twists can be found in [Tor04]). Looking at the local root number at 2 in such tables, we see that they change by $-1,1$ and -1 respectively, so we are in the condition of the last remark. To see whether we are in the principal series case or in the supercuspidal one, we can search for 
the curve in the quaternion algebra ramified at 2 and infinity. This can be done by choosing the correct order in such algebra (see HPS89]) and constructing ideal representatives for it in order to compute the Brandt matrices (see PS10] for an effective way to construct the ideals). It turns out that all four curves appear in such algebra (although it is clear that if one does, the others do as well); hence the component at 2 of all of them is supercuspidal.

Example 4.4. Consider the elliptic curve E3840c in Cremona's notation. Its quadratic twists by $\chi_{-1}, \chi_{2}$ and $\chi_{-2}$ are the curves E3840w, E3840n and E3840t respectively. Their local root numbers at 2 show that we are again in the condition of Remark 12. However, this curve does not show up in the quaternion algebra ramified at 2 ; hence it is principal series at 2 .

The last two examples show that both cases actually do occur, as was expected, and in particular proves that by considering only the variation of the local root number under twisting is not enough to determine the local factor at prime 2 .

\section{Some Remarks on Hilbert modular forms}

Although in all the previous sections we worked only with classical modular forms, the correspondence between Weil-Deligne representations and Hilbert modular forms works just as well. The properties/existence of the local root numbers do also, so we could just start with a Hilbert modular form over a totally positive number field $K$ in the first case. All the local computations are the same, but the problem is that there might be no global Hecke character $\chi$ to twist by. This comes from the fact that a totally positive number field $K$ (other than $\mathbb{Q}$ ) does have totally positive units different from 1 , which does not happen over $\mathbb{Q}$, so for a character $\chi_{\mathfrak{p}}$ to be well defined, it needs to be trivial at totally positive units.

To overcome this problem, starting from the character $\chi_{\mathfrak{p}}$, we choose an auxiliary prime $\mathfrak{q}$ which does not divide the level of the Hilbert modular form and such that $\chi_{\mathfrak{p}} \chi_{\mathfrak{q}}$ is trivial on totally positive units. Such primes always exist, since if $\chi_{\mathfrak{p}}$ is non-trivial on units, we can choose a basis $\left\{\nu_{1}, \ldots, \nu_{r}\right\}$ of the totally positive units such that $\chi_{\mathfrak{p}}\left(\nu_{1}\right)=-1$ and $\chi_{\mathfrak{p}}\left(\nu_{i}\right)=1$ for all $2 \leq i \leq r$. This is equivalent to saying that our prime $\mathfrak{p}$ is inert in the (ring of integers of the) quadratic extension $K\left[\sqrt{\nu_{1}}\right]$ and splits in the extension $K\left[\sqrt{\nu_{i}}\right]$, for $2 \leq i \leq r$. Then any prime $\mathfrak{q}$ with the same splitting behavior satisfies our hypothesis (and they always exist by Tchebotarev).

The behavior of twisting by $\chi_{\mathfrak{q}}$ is computed using the first case of Theorem 3.2. where we need to replace the quadratic symbol by $\chi_{\mathfrak{q}}(\pi)$ for $\pi$ a local uniformizer of $K_{\mathfrak{p}}$. In this way, we can extract the information needed to compute the local factor at $\mathfrak{p}$.

Example 5.1. Let $K=\mathbb{Q}[\sqrt{5}]$. The group of totally positive units is generated by the element $\left\langle\frac{3+\sqrt{5}}{2}\right\rangle$. Let $\mathfrak{P}_{31}=(6+\sqrt{5})$ be a prime ideal of norm 31 in $K$. In this case, $\chi_{\mathfrak{P}_{31}}\left(\frac{3+\sqrt{5}}{2}\right)=\chi_{31}(14)=1$, so no auxiliary prime is needed and everything works as over $\mathbb{Q}$. For example, consider the space of weight $(2,2)$ and level $\mathfrak{P}_{31}^{2}$ Hilbert modular forms. This can be computed using the Dembélé algorithm (see [Dem05]), which is implemented in Magma. It turns out that there are three forms having $\mathbb{Q}$ as a coefficient field. One of them is the twist of the elliptic curve of conductor $\mathfrak{P}_{31}$ given in [Dem08, Example 1]; hence both curves are Steinberg at the prime $\mathfrak{P}_{31}$. 
The other two curves are one twist of the other and have a Weierstrass equation:

$$
E: y^{2}+y=x^{3}-x^{2}-\left(\frac{7+3 \sqrt{5}}{2}\right) x
$$

(this equation was computed for us by Lassina Dembélé), and its twist has global minimal model:

$$
E_{\mathfrak{P}_{31}}: y^{2}+\sqrt{5} y=x^{3}-\left(\frac{1-\sqrt{5}}{2}\right) x^{2}-(639+285 \sqrt{5}) x-\left(\frac{4733+2113 \sqrt{5}}{2}\right) .
$$

If we compute the sign of their L-series, we see that $E$ has sign -1 while $E_{\mathfrak{P}_{31}}$ has sign +1 (actually using SAGE, [sag], one can check that $E$ has rank 1, while $E_{\mathfrak{P}_{31}}$ has rank 0 ). Since $\left(\frac{-1}{31}\right)=-1$, we conclude that $E$ is a principal series at $\mathfrak{P}_{31}$.

Example 5.2. Let $K$ be the totally real field of discriminant 257 obtained by adding to $\mathbb{Q}$ a root of the polynomial $t^{3}+2 t^{2}-3 t-1$ (it is not a Galois extension, since the discriminant is a non-square). The units in the ring of integer are generated by $\langle t, t-1\rangle$, with both of them having signatures $(-1,-1,1)$. The class number of $K$ is 1 , but the ray class number is 2 . The totally positive units are generated by $\left\langle t(t-1), t^{2}\right\rangle$. Let $\mathfrak{P}_{3}=\langle t+1\rangle$. It is a non-principal ideal for the ray class group (the sign of $t+1$ under the three embeddings are $(+,-,+)$ ). Also, $\chi_{\mathfrak{P}_{3}}(x(x-1))=\left(\frac{2}{3}\right)=-1$, so it does not define a global character. The space of parallel weight 2 forms of level $\mathfrak{P}_{3}^{2}$ has dimension 2 , and it splits into two eigenforms with rational coefficients (this space was computed to us by John Voight). One form is a twist of the other one by the narrow class character. One of the forms corresponds to the elliptic curve

$E: y^{2}+\left(t^{2}+3 t+3\right) x y+y=x^{3}+\left(t^{2}+t-1\right) x^{2}+\left(4 t^{2}+19 t+4\right) x+\left(4 t^{2}+9 t+2\right)$.

One way to prove that the curve is modular for the above modular form is to notice that the curve has torsion $\mathbb{Z} / 6 \mathbb{Z}$, so it is modular; and since there are no other forms in the space, it matches one of the two forms in our space (this argument and the equation for the elliptic curve is due to John Voight). Now we search for a prime ideal $\mathfrak{P}$ such that it has the same sign in the totally positive units as $\mathfrak{P}_{3}$. A small search reveals that the prime ideal $\mathfrak{P}_{7}=\langle 2 t+1\rangle$ satisfies the required property, since $\chi_{\mathfrak{P}_{7}}(t(t-1))=\left(\frac{-1}{7}\right)=-1$. So we can compute the twist of $E$ by the ideal $\mathfrak{P}_{3} \mathfrak{P}_{7}=\left\langle 2 t^{2}+3 t+1\right\rangle$. It is given by the equation (in global minimal model)

$$
\begin{aligned}
E_{\mathfrak{P}_{3} \mathfrak{P}_{7}}: y^{2}+(t+1) & x y=x^{3}+(t+1) x^{2} \\
+ & \left(-863 t^{2}-1791 t-442\right) x+\left(18919 t^{2}+40953 t+10179\right) .
\end{aligned}
$$

Its conductor has valuation 1 at the prime ideal $\mathfrak{P}_{3}$; hence both modular forms of level $\mathfrak{P}_{3}^{2}$ are Steinberg at $\mathfrak{P}_{3}$. Note that in this case, $\mathfrak{p}_{3}^{2}$ is the smallest conductor of any twist of the curve and has valuation 2 at $\mathfrak{p}_{3}$ (so it is not in any table computed before).

\section{ACKNOWLEDGEMEnTS}

The author would like to thank many people who during the last year helped in one way or another with this article: Gonzalo Tornaría, Tim Dokchitser, Lassina Dembélé, Victor Rotger, Luis Dieulefait, Mladen Dimitrov and John Voight. Lassina and John provided the examples in the last section and taught the author some 
useful things about computing with Hilbert modular forms. He would also like to thank the CRM in Barcelona for the pleasant time he spent there and for the nice working environment.

\section{REFERENCES}

[AL70] A. O. L. Atkin and J. Lehner. Hecke operators on $\Gamma_{0}(m)$. Math. Ann., 185:134-160, 1970. MR0268123(42:3022)

[AL78] A. O. L. Atkin and Wen Ch'ing Winnie Li. Twists of newforms and pseudo-eigenvalues of $W$-operators. Invent. Math., 48(3):221-243, 1978. MR.508986 (80a:10040)

[BR99] Pilar Bayer and Anna Rio. Dyadic exercises for octahedral extensions. J. Reine Angew. Math., 517:1-17, 1999. MR.1728550 (2001a:11191)

[Car86] Henri Carayol. Sur les représentations $l$-adiques associées aux formes modulaires de Hilbert. Ann. Sci. École Norm. Sup. (4), 19(3):409-468, 1986. MR870690 (89c:11083)

[Dav00] Harold Davenport. Multiplicative number theory, volume 74 of Graduate Texts in Mathematics. Springer-Verlag, New York, third edition, 2000. Revised and with a preface by Hugh L. Montgomery. MR1790423 (2001f:11001)

[Del73] P. Deligne. Les constantes des équations fonctionnelles des fonctions $L$. In Modular functions of one variable, II (Proc. Internat. Summer School, Univ. Antwerp, Antwerp, 1972), pages 501-597. Lecture Notes in Math., Vol. 349. Springer, Berlin, 1973. MR0349635(50:2128)

[Dem05] Lassina Dembélé. Explicit computations of Hilbert modular forms on $\mathbb{Q}(\sqrt{5})$. Experiment. Math., 14(4):457-466, 2005. MR2193808 (2006h:11050)

[Dem08] Lassina Dembélé. An algorithm for modular elliptic curves over real quadratic fields. Experiment. Math., 17(4):427-438, 2008. MR2484426 (2010a:11119)

[GK80] Paul Gérardin and Philip Kutzko. Facteurs locaux pour GL(2). Ann. Sci. École Norm. Sup. (4), 13(3):349-384, 1980. MR597744 (82i:22020)

[Hen79] Guy Henniart. Représentations du groupe de Weil d'un corps local, volume 2 of Publications Mathématiques d'Orsay 79 [Mathematical Publications of Orsay 79]. Université de Paris-Sud Département de Mathématique, Orsay, 1979. With an English summary. MR551495 (80m:12016)

[HPS89] Hiroaki Hijikata, Arnold K. Pizer, and Thomas R. Shemanske. The basis problem for modular forms on $\Gamma_{0}(N)$. Mem. Amer. Math. Soc., 82(418):vi+159, 1989. MR.960090 (90d:11056)

[Li80] Wen Ch'ing Winnie Li. On the representations of GL(2). I. $\varepsilon$-factors and $n$-closeness. J. Reine Angew. Math., 313:27-42, 1980. MR552460(81h:10042a)

[LW10] David Loeffler and Jared Weinstein. On the computation of local components of a newform. Math. Comp., 81(278):1179-1200 (2012). MR2869056 (2012k:11064)

[PS10] Ariel Pacetti and Nicolás Sirolli. Computing ideal class representatives in quaternion algebras. Submitted, arXiv:1007.2821, 2010.

[Rio06] Anna Rio. Dyadic exercises for octahedral extensions. II. J. Number Theory, 118(2):172188, 2006. MR2223979 (2007c:11132)

[Roh93] David E. Rohrlich. Variation of the root number in families of elliptic curves. Compositio Math., 87(2):119-151, 1993. MR.1219633 (94d:11045)

[sag] SAGE mathematics software, version 4.5.2. http://www.sagemath.org/.

[Tat79] J. Tate. Number theoretic background. In Automorphic forms, representations and Lfunctions (Proc. Sympos. Pure Math., Oregon State Univ., Corvallis, Ore., 1977), Part 2, Proc. Sympos. Pure Math., XXXIII, pages 3-26. Amer. Math. Soc., Providence, R.I., 1979. MR546607 (80m:12009)

[Tor04] Gonzalo Tornaría. Data about the central values of the l-series of (imaginary and real) quadratic twists of elliptic curves, http://www.ma.utexas.edu/users/tornaria/cnt/. 2004.

[Wei74] André Weil. Exercices dyadiques. Invent. Math., 27:1-22, 1974. MR0379445 (52:350)

Departamento de Matemática, Universidad de Buenos Aires, Pabellón I, Ciudad Universitaria, CP 1428, Buenos Aires, Argentina

E-mail address: apacetti@dm.uba.ar 